Int. J. Contemp. Math. Sciences, Vol. 2, 2007, no. 23, 1107 - 1120

\title{
A Bulk Service Queue with a Choice of Service and Re-Service under Bernoulli Schedule
}

\author{
A. Al-khedhairi and L. Tadj \\ Department of Statistics and Operations Research \\ College of Science, King Saud University \\ P.O. Box 2455, Riyadh 11451, Saudi Arabia \\ [akhediri, lotftadj]@ksu.edu.sa
}

\begin{abstract}
This paper investigates the queueing process of a bulk service queueing system under Bernoulli schedule. It also generalizes some known scenarios concerning the choice of service and re-service types. The queueing process is studied both in discrete time and in continuous time. Performance measures are derived and used to implement an optimal management policy of the system.
\end{abstract}

Mathematics Subject Classification: Primary 60K10, 60K25, secondary 90B22, 90B25

Keywords: Queue, quorum, Bernoulli schedule, vacation, re-service, optimal policy

\section{Introduction}

Queueing systems with server vacations are characterized by the fact that the idle time of the server may be used for other secondary jobs, for instance to serve customers in another system. Allowing servers to take vacations makes the queueing models more realistic and flexible in studying real-world queueing situations. Applications arise naturally in call centers with multi-task employees, customized manufacturing, telecommunication and computer networks, maintenance activities, production and quality control problems, etc. A wide class of policies for governing the vacation mechanism have been discussed in the literature. Comprehensive surveys on this topic can be found in Doshi $[11,12]$. In this connection, see also books by Takagi [30], Medhi [26], and the recent book by Tian and Zhang [31]. One of the fundamental objective of vacation models is to investigate the optimal control of a system in which a cost structure is assumed, see the recent survey of Tadj and Choudhury [28]. 
Our interest in this paper is in a class of queueing systems with vacation policy known as Bernoulli schedule. The classical vacation scheme with Bernoulli schedule discipline was introduced and studied by Keilson and Servi [14]. In their model, if on service completion the queue is not empty, the server goes on vacation with probability $p(p>0)$ and resumes service with probability $q=1-p$.

Our model also belongs to a class of systems where the service discipline involves more than one service and which has been receiving a lot of attention recently, see [2]-[4], [6]-[10], [13], [15]-[27], and [32]. Various scenarios have been considered in the literature thus giving rise to various denominations for this kind of systems. They are said to have an additional service channel or to have feedback, or to have optional re-service or to have two phases (or two types, or two stages) of heterogeneous service. Among the scenarios considered by the various authors, we cite the following:

Scenario 1: At the end of the first essential service a customer may either get the second optional service or depart from the system.

Scenario 2: The server provides two types of heterogeneous service and a customer may choose either type of service.

Scenario 3: A customer receives two phases of heterogeneous service one after the other then departs from the system.

Scenario 4: A customer chooses one of two types of heterogeneous service in a first phase, then he has the option to repeat or leave the system.

These scenarios have been combined with many of the different features known in queueing theory such as bulk arrival, server Bernoulli vacation schedule, server breakdowns, retrials, and some control policies such as N-policy and D-policy. The first work on queueing system with re-service is due to Madan [18] and then [19]. Madan [20] generalizes Madan [19] by allowing the binomial schedule instead of the Bernoulli schedule. In [21] Madan further generalizes its previous models by incorporating server vacations. Also, Madan and AlRawwash [23] and Madan et al. [24] allow the input process to be bulk instead of orderly. Besides Madan, other researchers have also been concerned with this type of queueing systems. For example, Kim and Chae [15] incorporate generalized vacations while Kim and Park [16] allow for threshold policies. Artalejo and Choudhury [2] take customers repeated attempts into consideration while Choudhury and Madan [8] incorporate N-policy. In addition, to N-policy Choudhury and Paul [9] cater for batch arrival. Finally, Wang [32] studies the case where the server may break down.

In the present paper, we incorporate bulk service and combine the above four scenarios into a single scenario, of which the four scenarios are special 
cases. We study the queueing process using the embedded Markov chain and semi-regenerative techniques. Finally we develop the total expected cost function per unit time and describe a procedure to determine some optimal system parameters.

\section{Model Description and Notation}

The bulk service queueing model with a choice of service and re-service under Bernoulli schedule described in this section is based on the following notation: Parameters:

$\left\{\begin{array}{lll}\lambda & : & \text { positive arrival rate of Poisson input process. } \\ p & : & \text { probability that the server takes a single vacation at a service completion epoch. } \\ b & : & \text { mean service time of a batch. } \\ v & : & \text { mean vacation time of the server. }\end{array}\right.$

Decision variables:

$$
\left\{\begin{array}{lll}
r & : & \text { threshold level. } \\
R & : & \text { maximum server capacity. }
\end{array}\right.
$$

Random variables:

$$
\left\{\begin{array}{lll}
B & : & \text { service time of a batch. } \\
V & : & \text { vacation time of the server. } \\
G & : & \text { required service time of a batch. }
\end{array}\right.
$$

Probability distribution functions (PDF):

$$
\begin{cases}B(t) & : \text { PDF of service time of a batch. } \\ V(t) & : \text { PDF of vacation time of the server. } \\ G(t) & : \text { PDF of required service time of a batch. }\end{cases}
$$

Laplaces-Stieltjes transforms (LST):

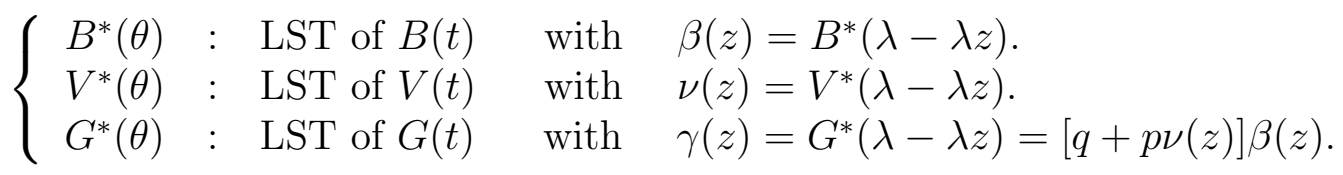

Stochastic processes:

$\left\{\begin{array}{lll}Q(t) & : & \text { number of customers in the system at an arbitrary instant of time } t . \\ Q_{n} & : & \text { number of customers in the system at the } n \text { service completion epoch. }\end{array}\right.$

Also, in what follows, for any random variable (rv) $X$, with $\operatorname{PDF} X(t)$ and LST $X^{*}(s)$, we will denote its first and second moments by $x^{(1)}$ and $x^{(2)}$. These two moments will be assumed to be finite if needed. Finally, we will 
denote by $P^{i}$ the conditional probability given $Q_{n}=i$, while $E^{i}$ will denote the corresponding conditional expectation.

Now consider a single-server queueing system with an orderly Poisson input with rate $\lambda>0$ and an infinite capacity waiting room. Let $r$ and $R$ be positive integers with $r \leq R$. Let $T_{n}$ represent the completion epoch of the $n$th service and let $Q(t)$ be the number of customers in the system at time $t$, so that $Q_{n}=Q\left(T_{n}^{+}\right)$is the number in the system at the $n$th service completion epoch. Service is in batches according to the following discipline:

- If $Q_{n} \geq r$, then service is provided to a group of customers of size $\min \left\{Q_{n}, R\right\}$. In a first phase, the group of customers has the option to choose either the first service type $B_{1}$ with probability $\alpha_{1}$ or the second service type $B_{2}$ with probability $\alpha_{2}=1-\alpha_{1}$. Once service of the first phase is finished, the group of customers has the option to leave the system, repeat the same service, or opt for the second type of service. If $\theta_{i j}$ is the probability to choose service type $i$ in the first phase and service type $j$ in the second phase, then with probability $\alpha_{i} \theta_{i j},(i=1,2 ; j=0,1,2)$, the service time $B$ of a group may be written as

$$
B=B_{i}+B_{j}
$$

with $B_{0}=0$. Note here that $\sum_{j=0}^{2} \theta_{i j}=1,(i=1,2)$. At the end of the second phase of service, the server has the option to take a single vacation of length $V$ with probability $p$ or serve the next group of customers (if any) with probability $q=1-p$. Thus, the service required for a group of units is

$$
G= \begin{cases}B+V, & \text { with probability } p, \\ B, & \text { with probability } q .\end{cases}
$$

- If $Q_{n}<r$, then the server becomes idle and waits until the number of units in the queue reaches the level $r$. Once this level reached, service is provided to a group of customers of size $r$.

Note that from relation (2.1), we have the LST of the modified service time

$$
B^{*}(s)=\sum_{i=1}^{2} \alpha_{i} B_{i}^{*}(s) \sum_{j=0}^{2} \theta_{i j} B_{j}^{*}(s),
$$

with $B_{0}^{*}(s)=1$, from which various moments can be deduced. For example, we find the first moment

$$
b^{(1)}=\left[\alpha_{1}\left(1+\theta_{11}\right)+\alpha_{2} \theta_{21}\right] b_{1}^{(1)}+\left[\alpha_{1} \theta_{12}+\alpha_{2}\left(1+\theta_{22}\right)\right] b_{2}^{(1)},
$$


and the second moment

$$
\begin{aligned}
b^{(2)}= & \alpha_{1}\left\{b_{1}^{(2)}+\theta_{11}\left[b_{1}^{(2)}+2\left(b_{1}^{(1)}\right)^{2}\right]+\theta_{12}\left[2 b_{1}^{(1)} b_{2}^{(1)}+b_{2}^{(2)}\right]\right\} \\
& +\alpha_{2}\left\{b_{2}^{(2)}+\theta_{21}\left[2 b_{1}^{(1)} b_{2}^{(1)}+b_{2}^{(2)}\right]+\theta_{22}\left[b_{2}^{(2)}+2\left(b_{2}^{(1)}\right)^{2}\right]\right\} .
\end{aligned}
$$

\section{Discrete Time Process}

3.1 Probability generating function Denote by $C_{n}$ the number of customer arrivals during the $n$th service. Then, the queueing process $\left\{Q_{n} ; n=\right.$ $0,1, \cdots\}$ is a Markov chain since it satisfies the following recursive formula:

$$
Q_{n+1}=\left(Q_{n}-R\right)^{+}+C_{n+1}
$$

where $f^{+}=\max \{f, 0\}$. Denote by $A$ the transition probability matrix (TPM) of $\left\{Q_{n}\right\}$ and by $A_{i}(z)=E^{i}\left[z^{Q_{n+1}}\right]$ the probability generating function (pgf) of the $i$ th row of $A$. Then,

$$
A_{i}(z)=z^{(i-R)^{+}} \gamma(z)
$$

Note from (3.1) that the TPM $A$ is a $\Delta_{R}$ matrix, see Abolnikov and Dukhovny [1] for related terminology. Using results from that paper, the Markov chain $\left\{Q_{n}\right\}$ is ergodic if and only if

$$
\left.\frac{d}{d z} A_{r}(z)\right|_{z=1}<1
$$

which is equivalent to

$$
\rho<1
$$

where

$$
\rho=\frac{\lambda\left(b^{(1)}+p v^{(1)}\right)}{R} .
$$

Introduce the pgf $p(z)=\sum_{i=0}^{\infty} p_{i} z^{i}$ where $p_{i}=\lim _{n \rightarrow \infty} P\left\{Q_{n}=i\right\}$. Under the condition (3.3), the equation $p(z)=\sum_{i=0}^{\infty} A_{i}(z) p_{i}$ along with the boundary condition $p(1)=1$ yield

$$
p(z)=\frac{\gamma(z) \sum_{i<R}\left(z^{R}-z^{i}\right) p_{i}}{z^{R}-\gamma(z)}
$$

Using a variant of Rouche's theorem, see also Abolnikov and Dukhovny [1], it can easily be shown that the unknown probabilities $p_{0}, \cdots, p_{R-1}$ are the 
solution of the following system of linear equations:

$$
\left\{\begin{aligned}
\sum_{i<R} \frac{d^{k}}{d z^{k}}\left[\gamma(z)-z^{i}\right]_{z=z_{s}} p_{i} & =0, \quad k=0,1, \cdots, k_{s}-1 ; \quad s=1,2, \cdots, S, \\
\sum_{i<R}(R-i) p_{i} & =R-\rho R
\end{aligned}\right.
$$

where $z_{s}$ are the roots of the characteristic equation

$$
z^{R}-\gamma(z)=0
$$

in the region $\bar{B}(0,1) \backslash\{1\}$ with their multiplicities $k_{s}$ such that $\sum_{s=1}^{S} k_{s}=R-1$.

3.2 Performance measures The system characteristics that can be derived in this section are as follows:

( $i$ ) The mean system size at a service completion epoch is given by

$$
L_{d}=\left.\frac{d}{d z} p(z)\right|_{z=1}=\frac{N^{\prime \prime}(1)-D^{\prime \prime}(1)}{2 D^{\prime}(1)}
$$

where

$$
\begin{aligned}
N^{\prime \prime}(1) & =2 \rho R(R-\rho R)+\sum_{i<R}[R(R-1)-i(i-1)] p_{i} \\
D^{\prime}(1) & =R-\rho R \\
D^{\prime \prime}(1) & =R(R-1)-\lambda^{2}\left[b^{(2)}+2 p b^{(1)} v^{(1)}+p v^{(2)}\right]
\end{aligned}
$$

(ii) Let $p=\left(p_{i}, i=0,1, \cdots\right)^{\top}$ and $\beta=\left(\beta_{i}, i=0,1, \cdots\right)^{\top}$ where $\beta_{i}=$ $E^{i}\left[T_{n+1}-T_{n}\right]$. Then, the stationary mean busy cycle $p \beta$ can be shown to satisfy

$$
p \beta=\sum_{i<r}\left(\frac{r-i}{\lambda}\right) p_{i}+b^{(1)}+p v^{(1)} .
$$

(iii) The system intensity defined by $\mathcal{I}=\lambda p \beta$ is given by

$$
\mathcal{I}=\sum_{i<r}(r-i) p_{i}+\rho R
$$

(iv) The server load $\ell_{n+1}$ on the $n$th cycle is given by

$$
\ell_{n+1}=\left\{\begin{array}{cc}
r, & Q_{n}<r \\
i, & r \leq Q_{n}<R \\
R, & Q_{n} \geq r
\end{array}\right.
$$


Therefore, it can easily be shown that the stationary mean server load $\ell=\sum_{i \geq 0} E^{i}\left[\ell_{n+1}\right] p_{i}$ satisfies

$$
\ell=(r-R) \sum_{i<r} p_{i}+\sum_{r \leq i<R}(i-R) p_{i}+R
$$

Using the boundary condition $p(1)=1$ and the above performance measures, it is easy to see that in the equilibrium, $\mathcal{I}=\ell$.

\section{Continuous Time Process}

4.1 Probability generating function The queueing process $\{Q(t) ; t \geq 0\}$ is readily seen to be semi-regenerative relative to the point process $\left\{T_{n} ; n=\right.$ $0,1, \cdots\}$ and $\left\{\left(Q_{n}, T_{n}\right) ; n=0,1, \cdots\right\}$ is a Markov renewal process. Introduce the pgf $\pi(z)=\sum_{i=0}^{\infty} \pi_{i} z^{i}$ where $\pi_{i}=\lim _{n \rightarrow \infty} P\{Q(t)=i\}$ are the steady-state system size probabilities. This stationary distribution exists under the same condition $\rho<1$. It can be determined using the main convergence theorem for semi-regenerative processes which, provided the probability distribution of the embedded Markov chain is known, gives much quicker result than the more popular method of supplementary variables. To use this theorem, we first compute the elements of the semi-regenerative kernel $K_{i j}(t)=P^{i}(Q(t)=$ $\left.j, T_{1}>t\right), i, j \in \mathbb{N}$, which are found to be

$$
K_{i j}(t)= \begin{cases}e^{-\lambda t} \frac{(\lambda t)^{j-i}}{(j-i) !}, & i \leq j<r, \\ \int_{0}^{t} \lambda e^{-\lambda(t-u) \frac{[\lambda(t-u)]^{r-i-1}}{(r-i-1) !} e^{-\lambda u} \frac{(\lambda u)^{j-r}}{(j-r) !}[1-G(u)] d u,} & i<r \leq j, \\ e^{-\lambda t} \frac{(\lambda t)^{j-i}}{(j-i) !}[1-G(t)], & r \leq i \leq j, \\ 0, & \text { otherwise. }\end{cases}
$$

We then successively evaluate the elements of the semi-integrated kernel $H=$ $\left(h_{i j} ; i, j \in \mathbb{N}\right)=\int_{0}^{\infty} K(t) d t$

$$
h_{i j}= \begin{cases}\frac{1}{\lambda}, & i \leq j<r \\ \int_{0}^{\infty} e^{-\lambda u} \frac{(\lambda u)^{j-r}}{(j-r) !}[1-G(u)] d u, & i<r \leq j \\ \int_{0}^{\infty} e^{-\lambda t} \frac{(\lambda t)^{j-i}}{(j-i) !}[1-G(t)] d t, & r \leq i \leq j \\ 0, & \text { otherwise }\end{cases}
$$


and the elements of the vector $h(z)=\left(h_{i}(z) ; i \in \mathbb{N}\right)$ where $h_{i}(z)$ is the generating function of the $i$ th row of matrix $H$

$$
h_{i}(z)= \begin{cases}\frac{1}{\lambda} \frac{z^{i}-z^{r} \gamma(z)}{1-z}, & i<r, \\ \frac{z^{i}}{\lambda} \frac{1-\gamma(z)}{1-z}, & i \geq r .\end{cases}
$$

Now by the main convergence theorem for semi-regenerative processes $\pi(z)=$ $\frac{p h(z)}{p \beta}$, along with equation (3.5) and equation (3.10), we obtain

$$
\pi(z)=\frac{1}{\mathcal{I}}\left\{\frac{1-z^{R}}{1-z} p(z)+\gamma(z) \sum_{i<R} \frac{z^{R}-\alpha_{i}(z)}{1-z} p_{i}\right\}
$$

where

$$
\alpha_{i}(z)= \begin{cases}z^{r}, & i<r \\ z^{i}, & r \leq i<R .\end{cases}
$$

4.2 Performance measures The system characteristics that can be derived in this section are as follows:

(i) The mean system size at an arbitrary instant of time is given by

$$
L_{c}=\left.\frac{d}{d z} \pi(z)\right|_{z=1}=L_{d}+\frac{R-1}{2},
$$

where $L_{d}$ is given by (3.8)

(ii) The mean idle period is given by

$$
I=\frac{1}{\lambda} \frac{\sum_{i<r}(r-i) p_{i}}{\sum_{i<r} p_{i}}
$$

This is because the probability that the server idles in the steady-state is given by

$$
\frac{\sum_{i<r} e_{\lambda, r-i}(t) p_{i}}{\sum_{i<r} p_{i}}
$$

where $e_{\lambda, k}(t)$ denotes the $k$-Erlang probability density function with parameter $\lambda$.

(iii) The mean busy period is given by

$$
B=\frac{1-\sum_{i<r} \pi_{i}}{\sum_{i<r} \pi_{i}} I
$$


This is because the probability that the server is idle in the steady-state is the same as the proportion of time he is idle in the long run:

$$
\sum_{i<r} \pi_{i}=\frac{I}{I+B} .
$$

(iv) The mean busy cycle is given by

$$
C=I+B
$$

\section{Optimal Policy}

The performance measures derived in the previous two sections can now be used to optimize the performance of the system. The design of an optimal management policy for a queueing system has received considerable attention, as shown by the survey conducted by Tadj and Choudhury [28]. This is known in queueing theory as the optimal control of the system. The aim is to find the the best values that the decision maker would implement in order to minimize the total expected cost per unit of time. Using a linear cost structure, this cost is given by

$$
T C(r, R)=c_{h} L_{c}+c_{o} \frac{B}{C}+c_{s} \frac{1}{C}+c_{a} \frac{I}{C}
$$

where

- $c_{h}$ : holding cost per unit time for each customer present in the system;

- $c_{o}$ : cost per unit time for keeping the server on and in operation;

- $c_{s}$ : setup cost per busy cycle;

- $c_{a}$ : startup cost per unit time for the preparatory work of the server before starting the service.

A similar cost structure was encountered by Tadj and Ke [29], and as in there, it is not possible to show that the cost function (5.1) is convex. The same algorithm proposed there can be used to determine the optimal values $\left(r^{*}, R^{*}\right)$. Denoting by

$$
R^{*}(r)=\min \{R \geq 1 \mid I(r, R)>0\},
$$

where

$$
I(r, R)=T C(r, R+1)-T C(r, R),
$$

we reproduce that algorithm here for convenience. 


\section{Algorithm 5.1}

Step 1. Set $r=1$. Determine $R^{*}(r)$ using (5.2) and compute $T C\left(r, R^{*}(r)\right)$ using (5.1).

Step 2. Compute $R^{*}(r+1)$ using (5.2) and $T C\left(r+1, R^{*}(r+1)\right)$ using (5.1).

Step 3. If $T C\left(r+1, R^{*}(r+1)\right)>T C\left(r, R^{*}(r)\right)$, STOP. The optimal values are $\left(r^{*}, R^{*}\right)=\left(r, R^{*}(r)\right)$. Otherwise, set $r=r+1$, GOTO Step 2.

\section{$6 \quad$ Numerical Illustration}

We present in this section a simple illustrative example by assuming that the service and vacation times are exponentially distributed. In this case

$$
\beta(z)=\frac{1}{1+\lambda b^{(1)}(1-z)}, \quad \nu(z)=\frac{1}{1+\lambda v^{(1)}(1-z)},
$$

and

$$
\gamma(z)=\frac{1+q \lambda v^{(1)}(1-z)}{\left[1+\lambda v^{(1)}(1-z)\right]\left[1+\lambda b^{(1)}(1-z)\right]}
$$

Also,

$$
b^{(2)}=2\left(b^{(1)}\right)^{2} \quad \text { and } \quad v^{(2)}=2\left(v^{(1)}\right)^{2} .
$$

The left-hand side of characteristic equation (3.7) is now a polynomial

$\lambda^{2} b^{(1)} v^{(1)} z^{R+1}-\left[\lambda^{2} b^{(1)} v^{(1)}+\lambda\left(b^{(1)}+v^{(1)}\right)\right] z^{R}+z^{R-1}+z^{R-2}+\cdots+z+1+q \lambda v^{(1)}=0$.

It is well known, see for example Chaudhry and Templeton [5], that this equation has $R-1$ simple roots $z_{s}, s=1, \cdots, R-1$ that belong to the unit ball $\bar{B}(0,1)$ in $\mathbb{C}$. The system of equations (3.6) becomes:

$$
\begin{cases}\sum_{i<R}\left[\frac{1+q \lambda v^{(1)}(1-z)}{\left[1+\lambda v^{(1)}(1-z)\right]\left[1+\lambda b^{(1)}(1-z)\right]}-z^{i}\right] p_{z=z_{s}} p_{i} & =0, \quad s=1, \cdots, R-1, \\ \sum_{i<R}(R-i) p_{i} & =R-\rho R .\end{cases}
$$

We implemented Algorithm 5.1 by taking the following system parameters $\lambda=1, b^{(1)}=0.8, v^{(1)}=0.3$, and $p=0.65$. The units costs were chosen as follows: $c_{h}=20, c_{o}=200, c_{s}=250, c_{a}=300$. The curve representing the total expected cost per unit of time, convex as expected, is shown in Figure 1. 


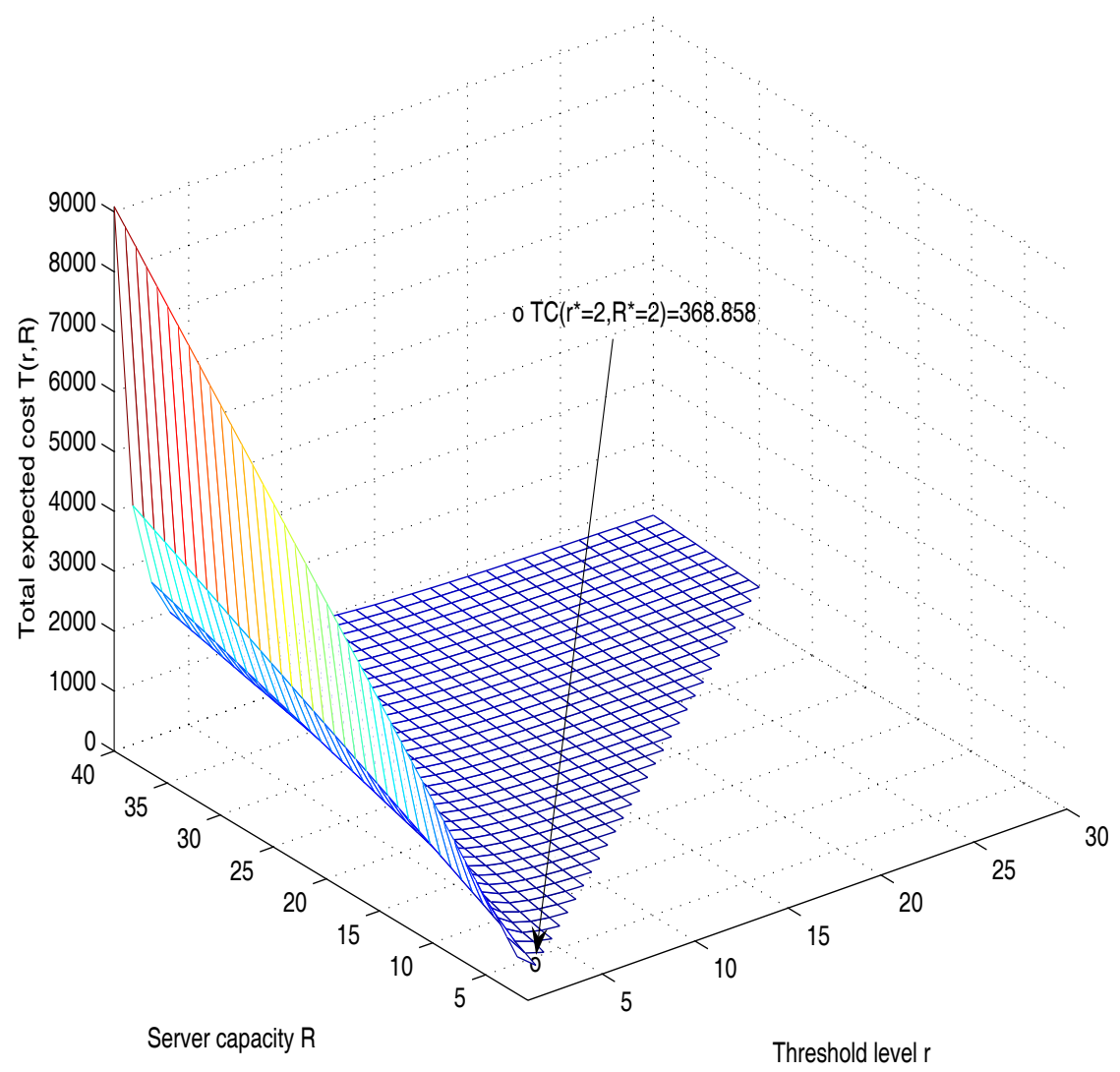

Figure 1. Variations of the total expected cost per unit of time.

The optimal values are found to be $r^{*}=2$ and $R^{*}=2$ for a minimum total cost $T C\left(r^{*}, R^{*}\right)=368.86$.

In summary, we have studied in this paper the queueing process of a bulk service queue under Bernoulli schedule. The group of customers being served has the option to choose either of two service types in a first phase. Once service of the first phase is finished, the group of customers has the option to leave the system, repeat the same service, or opt for the second type of service. Our model generalizes many of the models with optional re-service available in the literature. For this model we used the embedded Markov chain technique and a semi-regenerative approach to study the discrete and continuous time parameter queueing processes. We also derived various performance measures that were used to design an optimal management policy for the system. This study can be further generalized by including other known control policies such as the N, T, and/or D policy and by allowing the arrival process to be compound instead of orderly. 


\section{References}

[1] Abolnikov, L. and Dukhovny, A., Markov chains with transition delta matrix: Ergodicity conditions, invariant probability measures and applications, Journal of Applied Mathematics and Stochastic Analysis, 4:4 (1991) 333-356.

[2] Artalejo, J.R. and Choudhury, G. Steady state analysis of an M/G/1 queue with repeated attempts and two phase service, Quality Technology and Quantitative Management, 1:2 (2004) 189-199.

[3] Bertsimas, D. and Papaconstantinou, X. On the steady-state solution of the $\mathrm{M} / \mathrm{C}_{2}(a, b) / S$ queueing system, Transportation Sciences, 22 (1988) 125-138.

[4] Bertsimas, D. and Papaconstantinou, X. Analysis of the stationary $\mathrm{E}_{k} / \mathrm{C}_{2} / \mathrm{S}$ queueing system, European Journal of Operational Research, 37 (1988) 272-282.

[5] M.L. Chaudhry, M.L. and J.G.C. Templeton, A First Course in Bulk Queues, John Wiley, New York 1983.

[6] Choudhury, G. Some aspects of an M/G/1 queueing system with optional second service, TOP, 11:1 (2003) 141-150.

[7] Choudhury, G. and Madan, K.C. A two-phase batch arrival queueing system with a vacation time under Bernoulli schedule, Applied Mathematics and Computation, 149 (2004) 337-349.

[8] Choudhury, G. and Madan, K.C. A two-stage arrival queueing system with a modified Bernoulli schedule vacation under N-policy, Mathematical and Computer Modelling, 42 (2005) 71-85.

[9] Choudhury, G. and Paul, M. A batch arrival queue with an additional service channel under N-policy, Applied Mathematics and Computation, 156 (2004) 115-130.

[10] Choudhury, G. and Paul, M. Analysis of a two phases batch arrival queueing model with Bernoulli vacation schedule, Revista Investigatión Operacional, 25:3 (2004) 217-228.

[11] Doshi, B.T. Queueing systems with vacations: A survey, Queueing Systems, 1 (1986) 29-66.

[12] Doshi, B.T. Single-server queues with vacations, in: H. Takagi (Ed.) Stochastic Analysis of Computer and Communication Systems, NothHolland, Amsterdam (1990) 217-265. 
[13] Doshi, B.T. Analysis of a two-phase queueing system with general service times, Operations Research Letters, 10 (1991) 265-272.

[14] Keilson, J. and Servi, L.D. Oscillating random walk models for GI/G/1 vacation systems with Bernoulli schedule, Journal of Applied Probability, 23 (1986) 790-802.

[15] Kim, T.S. and Chae, K.C. Two-phase queueing system with generalized vacation, Journal of the Korean Institute of Industrial Engineers, 22 (1996) 95-104 (in Korean).

[16] Kim, T.S. and Park, A.Q. Cycle analysis of a two-phase queueing model with threshold, European Journal of Operational Research, 144 (2003) $157-165$.

[17] Krishna, C.M. and Lee, Y.H. A study of two-phase service, Operations Research Letters, 9 (1990) 91-97.

[18] Madan, K.C. A cyclic queueing system with three servers and optional two-way feedback, Microelectron. Rel., 28:6 (1988) 873-875.

[19] Madan, K.C. An M/G/1 queue with second optional service, Queueing Systems, 34 (2000) 37-46.

[20] Madan, K.C. On a single server queue with two stage general heterogeneous service and binomial schedule server vacations, The Egyptian Statistical Journal, 44 (2000) 39-55.

[21] Madan, K.C. On a single server queue with two stage general heterogeneous service and deterministic schedule server vacations, International Journal of System Science, 32 (2001) 837-844.

[22] Madan, K.C. and Abu Al-Rub, A.Z. On a single server queue with optional phase type server vacations based on exhaustive deterministic service and a single vacation policy, Applied Mathematics and Computation, 149 (2004) 723-734.

[23] Madan, K.C. and Al-Rawwash, M. On the $\mathrm{M}^{x} / \mathrm{G} / 1$ queue with feedback and optional server vacations based on a single vacation policy, Applied Mathematics and Computation, 160 (2005) 909-919.

[24] Madan, K.C., Al-Nasser, A.D., and Al-Masri, A.Q. On $\mathrm{M}^{[x]} /(\mathrm{G} 1, \mathrm{G} 2) / 1$ queue with optional re-service, Applied Mathematics and Computation, 152 (2004) 71-88.

[25] Medhi, J. A single server Poisson input queue with a second optional channel, Queueing Systems, 42 (2002) 239-242. 
[26] J. Medhi, Stochastic Models in Queueing Theory, (2nd ed.), Academic Press, Amsterdam 2003.

[27] Selvam, D.D. and Sivasankaran, V. A two-phase queueing system with server vacations, Operations Research Letters, 15 (1994) 163-168.

[28] Tadj, L. and Choudhury, G. (2005). Optimal design and control of queues, TOP, 13(1), 359-414.

[29] Tadj, L. and Ke, J-.C. Control policy of a hysteretic queueing system, Mathematical Methods of Operations Research, 57:3 (2003) 367-376.

[30] H. Takagi, Queueing Analysis: A Foundation of Performance Evaluation, Vacation and Priority Systems, Part I, North-Holland, New York 1991.

[31] N. Tian and Z.G. Zhang, Vacation Queueing Models: Theory and Applications, Springer, New York 2006.

[32] Wang, J. An M/G/1 queue with second optional service and server breakdowns, Computers and Mathematics with Applications, 47 (2004) 17131723.

\section{Received: February 7, 2007}

